\title{
TOTALIDAD Y TEORÍA DE CONJUNTOS
}

\section{TOTALITY AND SET THEORY}

\section{Roy Alfaro Vargas*}

\section{RESUMEN}

En este artículo se analiza el planteamiento de la teoría de conjuntos en detrimento de la noción de totalidad, como un medio para eliminar la división entre filosofía continental y la filosofía analítica. Además, se explica la actual implementación del conjunto vacío (Ø), como moda epistemológica en un sector de las actuales ciencias sociales y las humanidades, lo cual tiene como fin la construcción de un paradigma epistemológico que no solo muestra el agotamiento de la filosofía europea, sino que también produce ciencias incapaces de comprender la realidad y al contrario, crean discursos abstractos que legitiman patrones de consumo y políticas autoritarias.

PALABRAS CLAVE: CIENCIAS SOCIALES * HUMANIDADES * EPISTEMOLOGÍA * ALAIN BADIOU * SLAVOJ ŽIŽEK

\section{ABSTRACT}

This article analyzes the posing of set theory to the detriment of the notion of totality, as a means to annihilate the division between continental philosophy and analytical philosophy. Besides, it is explained the current implementation of the empty set (Ø) as an epistemological fashion into a sector of social sciences and humanities, which has as an end the construction of an epistemological paradigm that not only shows the exhaustion of European philosophy, but that also produces sciences that are unable of comprehending reality and, on the contrary, performs abstract discourses that legitimate consumption patterns and authoritarian policies.

KEYWORDS: SOCIAL SCIENCES * HUMANITIES EDUCATION * EPISTEMOLOGY * ALAIN BADIOU * SLAVOJ ŽIŽEK 


\section{INTRODUCCIÓN}

Se dice hoy que la filosofía occidental (europea) está "bajo el auspicio de un compromiso metafilosófico para la superación (o abandono) de la artificial, nociva y obsoleta división entre la aproximación 'continental' y la 'analítica' a la filosofía” (Gironi 2015, 1)리 lo cual se atestigua en "la explosiva oleada de un interés global por el trabajo de Alain Badiou" (Burhanuddin 2015, 1).

Tanto el abandono de la clásica separación entre filosofía continental (racionalista) y la analítica (empirista), como el auge del pensamiento badiouiano, tienen origen en los Écrits de Lacan (1966), quien introduce la categoría de conjunto como un medio para deshacerse de las consecuencias del concepto de totalidad: "La categoría de conjunto, para introducirlo, encuentra nuestra aprobación, en tanto que evita las implicaciones de la totalidad o las elimina" (Lacan 1966, 648).

Es así que a partir de esta percepción lacaniana surge la idea de implementar la noción de conjunto vacío (Ø) de la teoría de conjuntos, que permitiría, según los seguidores de esta idea, dejar de lado o abandonar el concepto de totalidad.

Con este breve marco, se reflexionará alrededor de la siguiente tesis: la eliminación de la noción de totalidad a favor del concepto de conjunto vacío de la teoría de conjuntos, conlleva reducir la totalidad, por un lado, a un proceso lógico-axiomático y por otro lado, enfatizar la parte sobre el todo, privilegiando la forma sobre el contenido; donde ambos señalamientos apuntan a consecuencias sobre el quehacer de las ciencias sociales y las humanidades.

\section{EL CONCEPTO DE CONJUNTO VACÍO}

Dicho de manera clara, "un conjunto es simplemente una lista de objetos, tal como $\mathrm{A}=\{\mathrm{a}, \mathrm{b}, \mathrm{c}, \mathrm{d}, \mathrm{e}\}$ o $\mathrm{B}=\{$ naranja, limón, lima, toronja, mandarina\}" (Smithson y Verkuilen 2006, 4). En términos de Badiou, "un conjunto está

1 Las traducciones de textos referidos en lengua alemana, francesa, inglesa, italiana y portuguesa, son propias. 'hecho de elementos', es la 'colección' [en mi lenguaje, el cuenta-por-uno] de sus elementos" (Badiou 2008, 62).

Dentro de esta perspectiva, el axioma de existencia de la teoría de conjuntos indica que, como punto de partida, "hay un conjunto (y este es el conjunto vacío)" (Gironi 2015, 24), lo cual quiere decir que "el vacío está incluido en cada conjunto" (Clemens 2005, 101), o como señala Badiou en Logiques des Mondes, "el vacío es un conjunto" (2006, 166), el cual "es el nombre propio del ser-en-tanto-ser" (Badiou 2006, 172), ya que, expresa Badiou en L'Être et l'événement, "el conjunto vacío es inextensional" $(1988,82)$, lo que quiere decir que no posee elementos.

Así, si el conjunto A, que se mencionó anteriormente, se conforma por los elementos $\{a, b, c, d, e\} ;$ esto quiere decir que el conjunto A es extensional, o sea, contiene elementos bien definidos diferentes del conjunto $\varnothing$. En otras palabras, hay una intensión lógica que define la propiedad de A; en este caso, el ser letras del alfabeto, lo que caracteriza a los elementos que incluye (su extensión) el conjunto A. Por otra parte, el conjunto vacío no tiene intensión y por ende, tampoco posee elementos, pues el vacío (lo Real lacaniano o la simple nada) no es definible: "Lo impresentable es inextensional" (Badiou 1988, 80).

Sin embargo, a pesar de que el conjunto vacío no tiene extensión, lo cierto es que "El conjunto vacío $\emptyset$ es el subconjunto de todo conjunto, incluso subconjunto de sí mismo" (Burhanuddin 2015, 46). Si se toma el conjunto $Z=\{1,2,3\}$, de este se puede derivar los siguientes subconjuntos: $\{1\},\{2\},\{3\},\{1,2\},\{2,3\},\{1,3\}$ $y$ además, el subconjunto $\{\varnothing\}$, el cero, que está contenido también en cada uno de los subconjuntos ya derivados, exempli gratia: $\{1, \varnothing\}$. De la misma manera, el conjunto $\emptyset$ contendría el subconjunto vacío, en cuanto "el conjunto vacío (...) es la unidad básica (el 'material') de la ontología de conjuntos" (Gironi 2015, 20) y en tanto todo conjunto "dispone, entre sus elementos, de un inexistente" (Badiou 2006, 340).

En este sentido, para Badiou, "el discurso del ser, ontológico, es un discurso matemático" (Minhot 2011, 30), en tanto "no hay 'el Uno' 
de la teoría de conjuntos, porque el conjunto de todos los conjuntos no existe" (Plotnitsky $2012,354)$ y por tanto, en esta perspectiva axiomática, no existe el Todo, sino lo múltiple que cuenta-por-uno. Así, "la propuesta de Badiou es que la ontología ejecute la garantía de la multiplicidad sin el uno" (Madarasz 2011, 118). No obstante, "lo múltiple no debe ser pensado aquí como multiplicidades de individuos, sino lo múltiple como previo a cualquier operación de estructura que genera unidades. Es una multiplicidad sin individuos y por ello solo puede ser matemática" (Minhot 2011, 30). Esta multiplicidad radica en que el conjunto $\emptyset$ a pesar de ser igual a sí mismo (principio de identidad de la lógica aristotélica), se encuentra además en todo conjunto.

Más simplemente dicho, si el conjunto $\emptyset$, como se señaló anteriormente, no tiene extensión y a la vez, este es el producto inmediato del axioma de existencia, así como, el subconjunto necesario de todo conjunto; entonces, "la teoría de conjuntos es una decisión ontológica que conlleva una formalización que le es propia” (Minhot 2011, 31), lo cual implica dentro del pensamiento de Badiou que "el vacío no es una producción del pensamiento, porque es desde su existencia que el pensamiento procede" $(2008,23)$ y que lo inextensional, lo irrepresentable, se resuelve "mediante lo que él llama una decisión de pensamiento, es decir, un axioma o un sistema axiomático" (Farrán 2013, 14) o lo que es lo mismo, el conjunto Ø "es así el alfa y el omega del sistema de Badiou" (Clemens 2005, 111).

La ontología de Badiou se define entonces como un sistema axiomático-matemático, anclado en la teoría de conjuntos, cuyo principio $y$ fin es el conjunto $\varnothing$, que funciona como una decisión de pensamiento que se enraíza en un puro cartesianismo (la separación entre res extensas y res cogitans, privilegiando esta última). Esta ontología busca "no estar limitada por ninguna jerarquía natural" (Lacan 1966, 648). Es decir, es una ontología desprovista de contenido empírico, sin extensión y con una intensión lógica vacía, que se distancia así de lo que en otros tiempos se llamó filosofía natural, es decir, la ciencia. Badiou, al igual que
Žižek, "refiere a la teoría de conjuntos (en la cual cualquier conjunto incluye el conjunto vacío como un elemento o subconjunto) con el fin de articular un espacio lógico" (Wood 2012, 61), lo cual implica que "para él, las ideas están ahí independientemente de, $y$ de antemano a, nosotros o nuestros símbolos" (Nirenberg y Nirenberg 2011, 589). De esta manera, "los axiomas de la teoría de conjuntos son para Badiou los axiomas de la ontología misma... La ontología es la "presentación de la presentación" (Gironi 2015, 27).

En otras palabras, "el Ser, para Badiou, forma una inconsistencia anterior y exterior a cualquier presentación consistente" (Burhanuddin 2015,23$)$, en tanto el conjunto $\emptyset$ tiene presencia en cuanto estructura lógica que es y que, en relación con el axioma de existencia, el conjunto $\emptyset$ tiene un rol fundador pero sin extensión (sin elementos), que adquiriría consistencia, ya que el $\emptyset$ no solo deriva como primer noción del axioma de existencia mismo, sino que además es parte (subconjunto) de cualquier otro conjunto. Así, el conjunto $\varnothing$ permite en Badiou presentar la estructura del Ser, como derivación del conjunto $\emptyset$, antes de presentar el Ser mismo, como consistencia (de hecho, hay una falacia de circularidad aquí, en cuanto premisa $y$ conclusión son una $y$ la misma cosa). Dicho de otro modo, dentro de la ontología badiouiana, "la impresentación ocurre bajo la fuerza presentativa del Axioma de Vacío" (Burhanuddin 2015, 101), es decir, "la ontología [para Badiou] solo puede hablar del Ser en sentido general, sustraída de cualquier particularidad" (Burhanuddin 2015, 103). Así, el conjunto $\varnothing$ deviene "una multiplicidad genérica [que] es una parte 'anónima' de este mundo, una parte que no corresponde a ningún predicado explícito" (Badiou 2006, 45) y en ese tanto "la filosofía, puesto que su categoría central es vacía, es esencialmente sustractiva" (Badiou 2002, 61).

La ontología sustractiva de Badiou tiene como fin alejarse del relativismo particularista de la postmodernidad; sin embargo, en cuanto el conjunto $\varnothing$ cuenta-por-uno, al ser la deducción primera del axioma de existencia, $y$ al ser el conjunto $\emptyset$, el subconjunto necesario de todo 
conjunto, esto lleva a fundar la ontología en el discurso particular(ista) de la matemática, lo cual no aleja a la propuesta de Badiou de una postmodernidad que se centra en el discurso (el giro lingüístico) y en un relativismo particularista. Para Badiou, "las matemáticas no presentan, en sentido estricto, nada” $(1988,13)$ $y$ estas remiten -en cuanto el conjunto $\emptyset$ permite generar conjuntos según la conveniencia de la decisión de pensamiento (el cual parece un sesgo de origen fenomenológico, ligado a la noción de intencionalidad) - a un lenguaje (el matemático) produciendo realidad, o sea, pensamiento creando un Ser-no-Ser, el vacío generando vacío à la Badiou.

Este vacío generador de vacío se justificaría, primero, en una falacia de circularidad (petitio principii) donde premisa y conclusión son la misma cosa (pura tautología) y segundo, en la necesidad que expresa la idea lacaniana de una teoría de conjuntos que "evita las implicaciones de la totalidad o las elimina" (Lacan 1966, 648). Badiou requiere pensar que "el ser es inmóvil" (2006, 378) y en consecuencia, poder afirmar que "el Todo no tiene ser" (Badiou 2006, 120), en cuanto el Todo, para Badiou, "no tiene evidencia física o fenomenológica" (2006, 121). Este planteamiento sostiene un argumento fundamental en Badiou: si no hay evidencia empírica (física o vivencial) del Todo; luego, no hay todo, sino algo que cuentapor-uno, es decir, el conjunto Ø: "uno de los principales conceptos cuestionados por los nuevos desarrollos matemáticos, es el de totalidad, no existe lenguaje axiomático total que legitime sus propios términos ...toda pretensión de totalidad auto-legitimada, de conformación del conjunto de todos los conjuntos, ha sido refutada por inconsistente" (Farrán 2013, 16-17).

Entonces, para Badiou (como para Lacan y Žižek que se anclan también alrededor del conjunto Ø), la totalidad no es deducible axiomáticamente. En otras palabras, para este paradigma cimentado en la formalización realizada a partir del conjunto $\varnothing$ no es posible crear un conjunto de todos los conjuntos, lo cual, según Badiou, Lacan y Žižek por ejemplo; anula la totalidad como forma. Sin embargo, ¿qué sucede con la totalidad como contenido? Es decir, ¿qué sucede si se piensa el Ser no como algo inmóvil (Badiou 2006), sino como movimiento? Sobre esto se discutirá más adelante.

\section{EL CONJUNTO Ø EN LAS CIENCIAS SOCIALES}

Como se ha observado, la asunción del conjunto $\emptyset$ tiene como fin el dejar de lado el concepto de totalidad, en el contexto de una operación lógico-axiomática donde el conjunto particular $(e l \emptyset)$ se interpreta por una decisión de pensamiento como contando-por-uno, a la vez que siempre es una parte de todo subconjunto. Finalmente, la parte se presenta como "la universalidad del 'no-Todo' en cuanto irresoluble antagonismo que caracteriza cada contenido particular del ser, volviéndolo ontológicamente incompleto" (Vighi 2014, 26).

Esto lleva a dos cuestiones; por un lado, se privilegia la abstracción en tanto se fundamenta en una ontología axiomática y axiomatizada, por otro, se da el establecimiento de sistemas que refieren "a un conjunto de cosas y las relaciones entre ellas" (Castellani y Hafferty 2009, 7; cursivas propias). Como se observa, ambos puntos (la política de la abstracción y la cuestión de los sistemas, modelos) más que relacionados, se funden en un mismo paradigma.

La política de la abstracción ${ }^{2}$ supone un proceso donde un todo se descompone en partes, para así analizarlas (Paolucci 2001a) y de hecho, donde "solo las parcialidades reciben análisis concreto" (Paolucci 2001b, 48), lo cual implica que "las partes de una totalidad pueden también ser conceptualizadas como totalidades en sí mismas" (Paolucci 2011, 58), es decir, la aplicación de esta idea conlleva que, por ejemplo, en El Capital de Marx, por ende, en la economía política, "la abstracción es la herramienta analítica esencial para identificar la estructura interna de las relaciones económicas capitalistas" (Callinicos 2014, 47). Así, la política de la abstracción lleva implícito que "la distinción entre lo abstracto $y$ lo concreto ${ }^{3}$...es

$2 \quad$ El lector puede profundizar más en el análisis de la política de la abstracción en Alfaro, 2013.

3 En esto se recuerda lo expresado por Marx, en los Grundrisse y que es negado por Callinicos: "el 
una entre conceptos" (Callinicos 2014, 131), lo cual se manifiesta como "el poder de la abstracción para construir un modelo de cómo trabaja la ingeniería económica del capitalismo" (Harvey 2014, 8; cursiva propia).

Asimismo, es claro que esta política de la abstracción se centra en el estudio de las partes, las cuales asume como un todo (whole), que al igual que en Badiou, cuenta-por-uno y que evade la totalidad, en cuanto "los todos (wholes) tienen una estructura definida; (mientras que) las totalidades no" (Bäck 2014, 224). Se crean entonces, a nivel teórico y desconectados de lo real-concreto, particulares abstractos, que son en este caso modelos explicativos axiomáticos que describen o interpretan cuestiones particulares. En palabras de Žižek (2014a), estos todos (wholes) engendran una totalidad (una universalidad) que se fundamenta en la división.

De este modo, con un lenguaje marxista y económico-político, la política de la abstracción estudia, en términos de la teoría de conjuntos, algunos subconjuntos (partes, wholes) que contienen elementos producto "de la progresiva introducción de determinaciones crecientemente complejas" (Callinicos 2014, 130), lo cual "no representa ninguna clase de corroboración empírica de las proposiciones individuales" (Callinicos 2014, 153). En otras palabras, la idea contenida en los Grundrisse de la relación entre lo abstracto y lo concreto, que permite confrontar la teoría (lo general) con un problema práctico, concreto (lo particular), como un medio para superar (aufheben) la teoría misma, en tanto aprehensión/construcción del movimiento mismo del objeto; se reduce a un juego de conceptos, donde para la política de la abstracción, "lo abstracto y lo concreto son un asunto de los lugares que los conceptos ocupen en el discurso" (Callinicos 2014, 132). Dentro de la política de la abstracción,

método de elevarse de lo abstracto a lo concreto es el único modo, para el pensamiento, de apropiarse de lo concreto, para reproducirlo como un concreto mental. De ningún modo, no obstante, el proceso de origen de lo concreto mismo" (Marx 1983, 35). el proceso se funda sobre una dinámica que se puede representar como: abstracto-más abstracto-más abstracto $y$ así en constante crecimiento de abstracción. Por su parte, en el pensamiento dialéctico la cuestión se define como: abstracto-concreto-abstracto o lo que dialécticamente es congruente, como: concreto-abstracto-concreto. Dicho más simplemente, ante un problema concreto que se intenta resolver, siempre se parte de un conocimiento previo (lo abstracto) con el cual se intenta resolver la situación planteada al enfrentar lo concreto. No obstante, como la realidad está en constante cambio, movimiento, lo abstracto (que se produjo en un tiempo anterior al problema concreto que se enfrenta) tiene desfases que, al ser estos visualizados en la práctica, obligan a replantear la teoría que buscaba comprender ese tipo de situaciones concretas, es decir, es la idea del "universal concreto".

Al contrario, en la política de la abstracción, el discurso que no refiere a lo concreto-real, se dirigiría a la construcción de un modelo (o sistema) cuyos elementos se caracterizarían dentro de la particularidad del modelo mismo, que es un conjunto de interrelaciones finalmente referidas a sí mismas y en tanto arreferenciales, son "nada". Dicho esto en términos más cercanos a Žižek, Badiou y Lacan (como ejemplo), "los modelos representacionales de la realidad imaginario-simbólica giran en torno a los irrepresentables antagonismos, conflictos, puntos muertos, callejones sin salida, etc., de lo Real" (Johnston 2014, 217). O sea, estos modelos inician y finalizan en el vacío $(\varnothing)$.

Dentro de este marco, el concepto de Web 3.0 de Fuchs (2008a y 2014) es muy esclarecedor con respecto a las implicaciones de esta política de la abstracción. De hecho, Fuchs (2008a) parte del análisis de los conceptos de Web 1.0 y Web 2.0, así como del análisis de las relaciones internas de cada uno de estos, para plantear el concepto de Web 3.0, la cual se definiría como un sistema de cooperación humana (Fuchs 2014), es decir, "las tecnologías de la Web 3.0 son tecnologías digitales en red que dan respaldo a la cooperación humana" 
(Fuchs 2008a, 127). Para este autor, la Web 3.0 es la superación (Aufhebung) del carácter cognitivo de la Web 1.0 y del énfasis comunicativo de la Web 2.0.

Desde el punto de vista de Fuchs y en el marco de aplicación de la política de la abstracción, la Web 3.0 parece un proyecto no solo interesante, sino también viable, más incluso cuando él apunta a ejemplos ya existentes (aunque de poco alcance) de redes sociales como Diaspora*, donde el usuario no es explotado como en Facebook (Fuchs 2014). No obstante, Fuchs, en su análisis, solo toma en cuenta las posibilidades de desarrollo de las redes sociales, sin percatarse del contexto económico-político que rodea el fenómeno de la actual Web 2.0 y lo cual implica que el cambio es total o no es cambio, ya que la parte (la Web 2.0 o la posible Web 3.0) está en función de la dinámica del actual capitalismo, el cual experimenta no solo al parecer una infranqueable incapacidad para hacer efectiva la plusvalía a nivel global (Harvey 2010), sino que cada vez más gira hacia extremismos de derecha (las amenazas estadounidenses a Rusia, Venezuela, etc., la pérdida del derecho a la protesta al modo de España, etc.). Dicho más claramente, la configuración de la Web 3.0 no radica en superar la Web 2.0, sino el capitalismo como un todo.

Queda claro que la Web 3.0 es un modelo, un conjunto con una dinámica específica $y$ un definido conjunto de relaciones internas, por lo que no solo se ancla en la política de la abstracción, sino que se fundamenta también en la noción de sistemas, que se vale de la teoría de conjuntos, en cuanto esta es - considerada como la mejor herramienta para la ciencia moderna según Castellani y Hafferty (2009) "el estudio de los medios propios para pensar, organizar y discutir la colección de objetos (conjuntos) y las relaciones que estos objetos (como conjuntos) tienen consigo mismos y con otros conjuntos" (Castellani y Hafferty 2009, 48). Finalmente, la política de la abstracción y la cuestión de los sistemas son lo mismo, la primera más cercana al vocabulario marxista y la segunda a la teoría de la complejidad ${ }^{4}$.

De esta manera, por ejemplo, Fuchs (2003 y 2008b) utiliza recursos propios de la teoría de la complejidad como los conceptos de auto-organización y de emergencia.

Fuchs afirma que "la teoría de la autoorganización como una teoría de los sistemas evolutivos propone la idea que el desarrollo de sistemas complejos no es totalmente accidental" (2003, 154). De hecho, la teoría de la autoorganización es un caso particular de la teoría de la complejidad social, la cual "es más una estructura conceptual que una teoría tradicional" (Castellani y Hafferty 2009, 34). Esta estructura conceptual termina por ser un conjunto de abstracciones colocadas en diferentes niveles, donde lo concreto, en tanto realidad y materialidad, desaparece; o sea, esta estructura conceptual es lo mismo que la política de la abstracción: conceptos relacionados en diferentes grados de abstracción.

Es claro que al igualar categorías y principios dialécticos con el metalenguaje de la teoría de la complejidad ${ }^{5}$, no se eliminan los sesgos de una teoría que crea sistemas complejos como simples modelos conceptuales, representacionales, amparados a su vez en la teoría de conjuntos y finalmente, en el conjunto $\emptyset$. La propuesta de Fuchs de la Web 3.0 reproduce el particularismo que deriva de la multiplicidad del conjunto $\varnothing$, debido a que es incapaz de relacionar y contextualizar adecuada $y$ dialécticamente el contexto total (lo cual implica ni empirismo en sí, ni racionalismo en sí, sino a veces uno $y$ otras veces el otro, según las necesidades derivadas de la praxis misma).

$4 \quad$ El lector puede consultar el texto de Byrne (1998), titulado Complexity Theory and the Social Sciences, que es un clásico en cuestiones de la teoría de la complejidad.

5 "El principio de emergencia corresponde a la categoría dialéctica de superación (Aufhebung) y a la negación de la negación, la categoría de parámetro de control, de puntos de bifurcación, de transición de fases, de no-linealidad, de selección, de fluctuación y de intensificación pueden ser tenidas como la reformulación del principio dialéctico de los cambios cuantitativos en cualitativos" (Fuchs 2008b, 114). 
El asunto aquí es sumamente complicado, pues con la implementación epistemológica de la teoría de conjuntos como modelo subyacente a todas las ciencias sociales, se llega a afirmar que "el positivismo está muerto" (Byrne 1998, 37), lo cual supondría que se acaba la tendencia positivista de dividir la realidad en parcelas (con una disciplina científica por cada parcela de la realidad) y el anclaje en el principio de identidad de la lógica formal (o sea, $\mathrm{A}=\mathrm{A}$ ), que imprime un sesgo cuya Weltanschauung (cosmovisión) se define de manera estática. Mas, a pesar de que los seguidores de la teoría de la complejidad (como tal y como política de la abstracción) creen que ellos tratan "con relaciones no-lineales, con cambios que no pueden ser encajados en una simple ley lineal" (Byrne 1998, 5); lo cierto es que esta propuesta simplemente ofrece "un conjunto de conceptos de trabajo ...para organizar sus investigaciones empíricas (ancladas en la parte, la cual asumen como un todo -whole-) dentro de la estructura y la dinámica de la mayoría de los sistemas sociales" (Castellani y Hafferty 2009: xii; paréntesis y cursiva propias). En otras palabras, la teoría de la complejidad como estructura conceptual sigue amarrada al principio lógico de identidad $(\mathrm{A}=\mathrm{A})$, que en palabras de Badiou significa que "el ser es inmóvil" (2006, 378), sentencia que deriva directamente de la asunción epistemo-ontológica de un cuerpo lógico-axiomático como la teoría de conjuntos, la cual solo proporciona "relaciones puramente abstractas entre conceptos abstractos" (Morrison 2015, 131) .

Del mismo modo, los estudios žižekianos de los medios de masas se ajustan a los imperativos del conjunto $\varnothing$ (lo Real lacaniano). Se parte en ellos de que "cuestiones acerca de cine, los medios y la cultura siempre se dirigieron a encontrar su camino dentro de los voluminosos volúmenes de escritos, conferencias y películas de Žižek" (Flisfeder

$6 \quad$ La política de la abstracción está igualmente presente en autores como: Krinsky (2013), Fineschi (2014), Smith (2014), Reuten (2014) y Moseley (2014). y Willis 2014, 3). Los autores ligados a los estudios žižekianos de los medios piensan que "ya que la realidad está estructurada como una ficción, una aproximación žižekiana al estudio de los medios dirige nuestra atención hacia las ficciones que ofrece lo Real" (Ibid, 11), en cuanto "lo Real es esa no-identidad, la imposibilidad de devenir 'sí-mismo', es ese algo que resiste la simbolización" (Hamza 2014, 34). En este marco, la implementación de lo Real (lacaniano) en el análisis de los medios, de la información en red y las tecnologías de la comunicación, plantea la paradójica necesidad, para estos žižekianos, de entender que "el ciberespacio no es suficientemente virtual" (Dean 2014, 213), es decir, lo Real crea un hueco, donde "los medios sociales ... son la manera en la cual el capitalismo ha tenido éxito en re-introducir la falta (lack) y la escasez dentro de un mundo de acceso instantáneo y de abundancia... Los medios sociales son una nueva frontera para el deseo" (Flisfeder 2014, 235-236). Así, lo Real lacaniano, en tanto eufemismo para el conjunto Ø, sume estos análisis en un marco epistemológico, que es incapaz de generar conocimiento, puesto que se articula en la falta, en esa nada que pretende generar nada, al modo de una especie de virtualidad que absorbe la realidad.

En el ámbito de la política, aparece el nuevo comunismo lacaniano, el cual se aglutina también alrededor del concepto de lo Real (el conjunto Ø) y que se define como:

un discurso abstraído que niega el carácter concreto de la problemática que se está enfrentando (pobreza, desvío de fondos públicos a lo privado, etc.) a través de divagaciones especulativas e idealistas de corte lacaniano, que dificultan o impiden asumir una acción política contestataria $y$ transformadora, que supere la realidad presente mediante la unidad de teoría $y$ práctica (Alfaro 2014a, 221).

De hecho, el nuevo comunismo lacaniano es asimismo un discurso que en tanto anclado en lo Real lacaniano, está fundamentado en el conjunto $\emptyset$, que conlleva una política del 
evento o más abiertamente, de la inacción, ya que no se puede actuar sobre la nada, sobre lo inextensional.

El post-postmodernismo de Nealon (2012), por su parte, "incita al desarrollo de una lógica cultural válida y funcional para el just-in-time capitalism (el capitalismo de sololo-justo)" (Alfaro 2014b, 104), donde se asume "la homología entre la lógica cultural de la globalización y la lógica económica del capital financiero" (Nealon 2012, 32). En este sentido, las humanidades, al igual que el capital financiero, se deben mover hacia la creación de mercancías que simplemente sean consumibles, sin importar la calidad y su ligamen con el mundo y la economía real ${ }^{7}$. Se puede afirmar que "el post-postmodernismo ...es un todo vacío" que se enlaza "con el 'evento' de Badiou” (Alfaro 2014b, 106).

\section{DEL CONJUNTO $\varnothing$ A LA TOTALIDAD}

Hasta aquí se tiene un conjunto $\varnothing$ que, en tanto deducible del axioma de existencia $y$ en cuanto este es lo único constante como subconjunto de todo conjunto (o sea, contandopor-uno y seguidamente, como multiplicidad), permite plantear conjuntos, modelos, sistemas, estructuras conceptuales o como se les quiera denominar. Estos serían conjuntos planteados a conveniencia por el principio de decisión del pensamiento y que, cualesquiera que sean los elementos del conjunto planteado dentro de una investigación científica y académica, estos conjuntos se validarían (más allá de la ocurrencia que se plantee) por ya contener el conjunto $\emptyset$. Así, en una suerte de falacia de circularidad, el conjunto $\varnothing$ es la causa y el efecto de la existencia conceptual $y$ de la validez de cualquier planteamiento científico, en este caso, las ciencias sociales $y$ de hecho, en las humanidades. $\mathrm{O}$ sea, para la ontología axiomática de Badiou, el conjunto Ø (lo Real) es una imposibilidad y una necesidad (Žižek 2014b). Imposible porque el

$7 \quad$ Se podría decir que el fenómeno de la erróneamente denominada ciencia ficción costarricense es una manifestación, muy folklórica, de esta lógica; pero, esto requiere una investigación y una reflexión más allá de los límites de este escrito. conjunto $\emptyset$ es solo un concepto sin extensión, es decir, nada; $y$ necesario porque es el punto indispensable de partida para cimentar un nihilismo onto-epistemológico, donde Badiou propone "una disolución de la materia" (Burhanuddin 2015,12 ).

Disolución de la materia que se manifiesta, dentro del actual contexto, en la creencia de que "la naturaleza abstracta de la matemática puede, no obstante, producir información física" (Morrison 2015, 4). Al estilo (post)postmoderno, pero con el lenguaje matemático derivado y dependiente del lenguaje natural ${ }^{8}$, la ontología axiomática badiouiana basada en la teoría de conjuntos se atribuye la capacidad de crear realidad. De este modo, la forma lógica produciría, de acuerdo con Badiou, la realidad, en función de alguna clase de solipsismo racionalista $y / 0$ racionalizado; a la vez que anclado en el estudio de la parte (o el nuevo todo whole-) cumpliría con los requerimientos del empirismo. Así, se está ante un paradigma que aglutina dos puntos contradictorios: un racionalismo prácticamente solipsista y un empirismo sensualista, lo cual permitiría, para los que profesan la fe en el conjunto $\emptyset y$ en tal ontología axiomática:

moverse entre el empirismo y el racionalismo a través de un simultáneo mandato (científicamente negociado $y$ conceptualmente mediado) de la sensibilidad como una fuente de conocimiento del mundo exterior y de la capacidad de la razón humana, para ir más allá de la percepción sensible, en un discernir secuestrante de las estructuras ocultas de la realidad (Gironi 2015, 36), [que al igual que] toda la física moderna trata solo con modelos idealizados (Plotnitsky 2015, 559).

Sin embargo, la cuestión alrededor de la teoría de conjuntos, en general y del conjunto $\emptyset$, en particular, tiene sus restricciones:

\footnotetext{
8 De hecho, la noción del conjunto $\emptyset$ rompe el principio de que "todo lenguaje es referencial" (Gaskin 2013, 290).
} 
$\diamond \quad$ "Si nosotros somos lectores lógicos más que unos dogmáticos, nosotros nos detendremos a notar que no hay nada necesario acerca de tal origen ex nihilo. Asumir la existencia del conjunto vacío como un axioma es meramente una escogencia técnicamente conveniente" (Nirenberg y Nirenberg 2011, 590-591).

$\diamond \quad$ El carácter formal-axiomático (el concepto mismo de vacío, la nada) de la teoría de conjuntos no soporta la confrontación con el contenido de la realidad, el cual sobrepasa la inerte formalización. El vacío y la nada son solo eso, conceptos; de hecho, el vacío y la nada siempre son algo, porque la realidad, el Ser, no es inmóvil, lo cual requiere del concepto de totalidad que "posibilita la superación de las visiones parceladas $y$ fragmentadas del proceso social" (Torres 2011, 305).

El hecho de que la totalidad no sea empíricamente perceptible $y$ racionalmente abarcable, no es razón para hacerla irreal, más cuando se entiende que por su misma condición de ser cambiable, transformable, móvil, tanto el empirismo en sí, como el racionalismo en sí son herramientas nada útiles para la comprensión (Verstehen) de la totalidad, la cual "solo se revela fecundamente a aquel que la considera dialécticamente" (Lefebvre 2011, 112), entendiendo que "la totalidad ...es histórica (es decir, práctica y real)" (Lefebvre 1970, 24). De esta manera, la totalidad al ser práctica y real, pone de frente a un elemento que el abstraccionismo de la ontología axiomática derivada de la teoría de conjuntos perdió, o sea, lo concreto.

Lo concreto, dice Marx (1983), está multideterminado, siendo así más rico que la abstracción discursiva que se tenga de lo concreto mismo en un punto discontinuo de la continuidad de tal concreto, que se manifiesta en tiempo presente $y$ responde a un imperativo práctico. Lo concreto es el "contenido" desenvolviéndose en diferentes formas desde un estado "real" (del Ser) a uno "posible" (del Deber Ser), en cuanto el "objeto" y el "sujeto" condensan la totalidad en su relación dialéctica, en la praxis.
En lo concreto, lo "universal" de lo abstracto, se somete a prueba ante lo "particular" del objeto de estudio concreto, que obliga a redefinir lo abstracto, la teoría, el discurso, para adecuarlo a una nueva concretez que, como movimiento que es (como entropía), siempre supera lo abstracto, o sea, la teoría que pretendía explicar lo concreto en sí. La suposición de que $\mathrm{A}=\mathrm{A}$, en todos los casos, se derrumba ante la noción de congruencia $(\mathrm{A} \mathrm{A})^{9}$, donde lo que rige no es la identidad, sino la identidad y la diferencia, que hacen que Ser y pensamiento (el lenguaje axiomático, por ejemplo), como relación de lo concreto y lo abstracto, se adecuen mutuamente, en tanto la identidad cambia con el tiempo, la cual inserta la diferencia que se supera en una nueva identidad.

La totalidad implica que el Ser es móvil, cambiante en sí y para sí, por su propia entropía y por la praxis humana. La Web 3.0 de Fuchs es posible como producto de la acción humana, pero requiere un cambio en las condiciones objetivas del sistema, como un todo. Si la gente migrara de Facebook (que explota al usuario de esta red) a Diaspora* (que es una red no-comercial); entonces, el capital también migraría junto con la gente, ya que esto es una necesidad del capitalismo como un todo. El anclarse en la parte (el whole derivado de la aplicación de la teoría de conjuntos) lleva a la nada la propuesta de Fuchs. Dicho en otros términos, lo concreto no es una relación entre conceptos, sino la relación sujeto-objeto dentro de la necesidad de resolver el problema que se enfrenta en algún determinado momento, a través de lo contingente de tal relación.

\section{CONCLUSIÓN}

Es evidente que la eliminación del concepto de totalidad a favor de la noción de conjunto $\varnothing$, conlleva reducir la totalidad misma a un proceso lógico-axiomático, con el consecuente énfasis en la parte (whole) sobre el todo, lo cual implica privilegiar de manera absoluta la forma sobre el contenido.

$9 \quad$ El lector puede confrontar Kojève (2013), donde se explica la noción de congruencia. 
El intento de superar la división entre filosofía continental y filosofía analítica ha llevado a la filosofía europea al callejón sin salida del nihilismo onto-epistemológico del conjunto $\emptyset$; lo cual, mirando la crisis social, política $y$ económica que vive Europa, muestra el "agotamiento" del pensamiento filosófico europeo, que se ha rendido ante los imperativos reaccionarios del neoliberalismo.

La noción de conjunto Ø lleva a las ciencias sociales $y$ a las humanidades ( $y$ ciertamente también a la matemática y la física —en su faceta mecánico-cuántica ${ }^{10-}$ ) a convertirse en simples discursos que legitiman todo aquello que se puede consumir, desde la más simple mercancía hasta el más elaborado proceso identitario (post)postmoderno.

El conjunto $\emptyset$ remite a un empirismo sin extensión y a un racionalismo sin intención; luego, se tiene lo peor de cada una de estas propuestas filosóficas, lo cual tiene serias implicaciones éticas, ya que despóticamente, en lo político por ejemplo, se podría crear un conjunto denominado Irak, cuyos elementos sean \{posesión de armas nucleares, amenaza a la seguridad nacional estadounidense, $\varnothing\}$, donde el conjunto vacío, por mera decisión axiomática, legitima la "Realidad" del conjunto completo, al ser el conjunto $\emptyset$ parte del conjunto Irak y por ser derivación y expresión múltiple del axioma de existencia. Luego, el conjunto Irak sería una verdad axiomática (más allá de la falsedad de sus elementos) y las consecuencias de esto ya las conocemos.

El conjunto $\varnothing$ como base onto-epistemológica ( $y$ cultural en lo que respecta a $\mathrm{Eu}-$ ropa) que rompe con la distinción entre filosofía analítica y filosofía continental, es insostenible, debido a que somete el quehacer de las ciencias sociales y las humanidades a dos elementos ya superados en la historia de la filosofía: el nihilismo y el solipsismo. La nada no puede ser el fundamento de la existencia que en sí misma niega esa nada rotundamente $y$, como pasa con todo idealismo filosófico, la realidad no se genera a

10 Confróntese: Žižek (2014a y 2014b) quien se vale de la mecánica cuántica para introducir también el relativismo y el nihilismo, en su reflexión. partir de la conciencia, aunque esta se quiera ver como algo objetivo, fuera y más allá del ser humano, es decir, como teoría de conjuntos.

La totalidad, como noción práctico-real y como categoría filosófica, al contrario, permite dialécticamente asumir no solo la forma lógica, el pensamiento, sino también el contenido (el proceso histórico, la entropía y el movimiento). La totalidad recompone la praxis que la ontología axiomática de Badiou (Lacan, Žižek y todos los que se amparan en lo Real lacaniano) destruye, al restablecer la relación entre sujeto $y$ objeto, como praxis social $y$ transformadora.

\section{BIBLIOGRAFÍA}

\section{LIBROS}

Bäck, Allan. 2014. Aristotle's Theory of Abstraction. Heidelberg: Springer.

Badiou, Alain. 2008. Number and Numbers. Cambridge y Malden (MA): Polity.

Badiou, Alain. 2006. Logiques des mondes. L'Être et l'événement, 2. París: Éditions du Seuil.

Badiou, Alain. 2002. Condiciones. México: Siglo XXI Editores.

Badiou, Alain. 1998.Court traité d'ontologie transitoire. París: Éditions du Seuil.

Badiou, Alain. 1988. L'Être et l'événement. París: Éditions du Seuil.

Burhanuddin, Baki. 2015. Badiou's Being and Event and the Mathematics of Set Theory. Londres y Nueva York: Bloomsbury.

Byrne, David. 1998. Complexity Theory and the Social Sciences. An Introduction. Londres y Nueva York: Routledge.

Callinicos, Alex. 2014. Deciphering Capital. Marx's Capital and its Destiny. Londres: Bookmarks Publications.

Castellani, Brian y Frederic William Hafferty. 2009. Sociology and Complexity Science. A New Field of Inquiry. Heidelberg: Springer.

Dean, Jodi. 2014. "The Real Internet”. En Žižek and Media Studies. A Reader, editado por Mattew Flisfeder y Louis-Paul Willis. Nueva York. Palgrave McMillan: 221-227. 
Fineschi, Roberto. 2014. "On Hegel's Methodological Legacy in Marx". En Marx's Capital and Hegel's Logic. A Reexamination, editado por Fred Moseley y Tony Smith. Leiden y Boston. Brill: 140-163.

Flisfeder, Mattew y Willis, Louis-Paul. 2014. "Introduction. Žižek and Media Studies, Beyond Lacan”. En Žižek and Media Studies. A Reader, editado por Mattew Flisfeder y Louis-Paul Willis. Nueva York. Palgrave McMillan: 1-12.

Flisfeder, Mattew. 2014. "Enjoying Social Media”. En Žižek and Media Studies. A Reader, editado por Mattew Flisfeder $y$ Louis-Paul Willis. Nueva York. Palgrave McMillan: 229-240.

Fuchs, Christian. 2014. Social Media. A Critical Introduction. Los Angeles, CA: Sage.

Fuchs, Christian. 2011. Foundations of Critical Media and Information Studies. Londres y Nueva York: Routledge.

Fuchs, Christian. 2008a. Internet and Society. Social Theory in the Internet Age. Nueva York y Londres: Routledge.

Gaskin, Richard. 2013. Language, Truth, and Literature. A Defense of Literary Humanism. Oxford: Oxford University Press.

Gironi, Fabio. 2015. Naturalising Badiou. Mathematical Ontology and Structural Realism. Londres y Nueva York: Palgrave Macmillan.

Hamza, Agon. 2014. "The Sublime Absolute: Althusser, Žižek, and the Critique of Ideology". En Žižek and Media Studies. A Reader, editado por Mattew Flisfeder y Louis-Paul Willis. Nueva York. Palgrave McMillan: 27-38.

Harvey, David. 2014. Seventeen Contradictions and the End of Capitalism. Nueva York: Oxford University Press.

Harvey, David. 2010. The Enigma of Capital and the Crisis of Capitalism. Londres: Profile Books.

Johnston, Adrian. 2014. Adventures in Trascendental Materialism. Dialogues with Contemporary Thinkers. Edinburgh: Edinburgh University Press.
Krinsky, John. 2013. "Marxism and the Politics of Possibility: Beyond Academic Boundaries". En Marxism and Social Movements, editado por Colin Barker, Laurence Cox, John Krinsky y Alf Gunvald Nielsen. Leiden y Boston: Brill: 103-121.

Lacan, Jacques. 1966. Écrits. París: Éditions du Seuil.

Lefebvre, Henri. 1970. La fin de l'histoire. Épilégomènes. París: Les Éditions de Minuit.

Marx, Karl. 1983. Grundrisse der Kritik der politischen Ökonomie (MEW Band 42). Berlín. Dietz.

Morrison, Margaret. 2015. Reconstructing Reality. Models, Mathematics, and Simulations. Nueva York: Oxford University Press.

Moseley, Fred. 2014. "The Universal and the Particulars in Hegel's Logic and Marx's Capital". En Marx's Capital and Hegel's Logic. A Reexamination, editado por Fred Moseley y Tony Smith. Leiden $y$ Boston. Brill: 115-139.

Nealon, Jeffrey T. 2012. Post-postmodernism or, The Cultural Logic of Just-in-Time Capitalism. Standford, CA: Standford University Press.

Paolucci, Paul. 2011. Marx and the Politics of Abstraction. Leiden y Boston: Brill.

Plotnitsky, Arkady. 2015. "Reality, Causality, and Probability, from Quantum Mechanics to Quantum Field Theory". En Quantum Foundations and Open Quantum Systems. Lecture Notes of the Advanced School, editado por Theo M. Nieuwenhuizen, Claudia Pombo, Claudio Furtado, Andrei Yu Khrennikov, Inácio A Pedrosa y Václav Špička. Singapur: World Scientific Publishing: 521-603.

Reuten, Geert. 2014. "An Outline of the Systematic-Dialectical Method: Scientific and Political Significance". En Marx's Capital and Hegel's Logic. A Reexamination, editado por Fred Moseley y Tony Smith. Leiden y Boston. Brill: 243-268. 
Rodman, Gilbert B. 2015. Why Cultural Studies? Malden (MA)-Oxford: WileyBlackwell.

Smith, Tony. 2014. "Hegel, Marx and the Comprehension of Capitalism". En Marx's Capital and Hegel's Logic. A Reexamination, editado por Fred Moseley y Tony Smith (eds.). Leiden y Boston. Brill: 17-40.

Smithson, Michael y Jay Verkuilen. 2006. Fuzzy Set Theory. Applications in the Social Sciences. Thousand Oaks (CA): Sage.

Wood, Kelsey. 2012. Žižek: A Reader's Guide. Wiley-Blackwell.

Žižek, Slavoj. 2014b. Absolute Recoil. Towards a New Foundation of Dialectical Materialism. Londres y Nueva York: Verso.

Žižek, Slavoj. 2014a. Event. A Philosophical Journey Through a Concept. Brooklyn y Londres: Melville House.

PUBLICACIONES PERIÓDICAS

Alfaro Vargas, Roy. 2014a. "El nuevo comunismo lacaniano". Revista de Ciencias Sociales 144, n. . II: 211-223.

Alfaro Vargas, Roy. 2013. "Paul Paolucci y la política de la abstracción”. Endoxa: series filosóficas 32: 207-227.

Alfaro Vargas, Roy. 2014b. "Postpostmodernismo". Revista Reflexiones 93, n. ${ }^{\circ} 2: 103-113$.

Clemens, Justin. 2005. "Doubles of Nothing: The Problem of Binding Truth to Being in the Work of Alain Badiou". Filozofski Vestnik 2, n XXVI: 97-111.

Farrán, Roque. 2013. "Badiou y Lacan: algunas consideraciones en torno a lo real, la ontología y el concepto de sujeto en la práctica filosófica y psicoanalítica”. El laberinto de arena 1, n. $^{\circ}$ 1: 1-28.

Fuchs, Christian. 2008b. "Dialektisches Denken als Grundlage der Kritik des transnationalen informationellen Kapitalismus". Vorschein 30: 97-119.

Fuchs, Christian. 2003. "Structuration Theory and Self-Organization". Systemic
Practice and Action Research 2, n. ${ }^{\circ} 16$ : 133-167.

Kojève, Alexandre. 2013. "¿Qué es la dialéctica?" Revista de Ciencias Sociales 139, n. ${ }^{\circ}$ I: 91-102.

Lefebvre, Henri. 2011. "La noción de totalidad en las ciencias sociales". Telos. Revista de estudios interdisciplinarios en Ciencias Sociales 1, n. ${ }^{\circ}$ 13: 105-124.

Madarasz, Norman R. 2011. "A Superação da Ontologia Fundamental de Heidegger pela Filosofia em Sistema de Alain Badiou”. Ensaios Filosóficos IV: 93-119.

Minhot, Leticia O. 2011. "Teoría de conjuntos y ontología”. Arbor Ciencia, Pensamiento y Cultura 187. 25-32.

Nirenberg, Ricardo L. y David Nirenberg. 2011. "Badiou's Number: A Critique of Mathematics as Ontology". Critical Inquiry 37: 583-614.

Paolucci, Paul. 2011a. "Assumptions of the Dialectical Method". Critical Sociology 3, n. ${ }^{\circ} 27: 116-146$.

Plotnitsky, Arkady. 2012. "Experimenting with ontologies: sets, spaces, and the topos with Badiou and Grothendieck". Environment and Planning D: Society and Space 30: 351-368.

Torres, Esteban. 2011. "Cambio social y totalidad". Cinta de Moebio 42: 302-312.

Vighi, Fabio. 2014. "Dall'evento al sintomo: Badiou e l'ontologia lacaniana". Badiou Studies 1, n. 3: 24-47.

TESIS

Paolucci, Paul. 2001b. "Dialectical Methodology, Power \& Capital: Dialectical Methods, Foucault's Encounter with Marxism, and Techniques of Class Domination into the Global Era”. Tesis doctoral de Filosofía. Universidad de Kentucky (USA).

Fecha de ingreso: 25/03/2015 Fecha de aprobación: 11/01/2016 